\title{
La RSE : un langage commun entre entreprise classique et entreprise sociale
}

\author{
EL GHMARI Imad ${ }^{1}$ et EL MOUSSAID Mohammed Amine ${ }^{2}$ \\ ${ }^{1}$ Faculté des Sciences Juridiques, Economique et sociale - Souissi, Université Mohamed V. Rabat, Maroc \\ Laboratoire : recherche en management des organisations, droit des affaires et développement durable \\ ${ }^{2}$ Faculté des Sciences Juridiques, Economique et sociale - Fès, Université Sidi Mohamed Ben Abdellah \\ Fès, Maroc \\ Laboratoire : interdisciplinaire de recherche en économie, finance et management des organisations
}

\begin{abstract}
Résumé : La Responsabilité sociale des Entreprises (RSE) est une notion complexe qui s'est largement développée dans le monde entier. Connu depuis son origine éthique en Amérique, le concept a évolué pour inclure d'autres aspects économiques, managériaux, environnementaux, sociaux et politiques. Le fruit de cette évolution est une notion complexe qui ne se pratique pas de la même manière dans les pays du monde, compte tenu des différents facteurs environnementaux tels que la culture, les valeurs éthiques, la vision, la finalité de la nation ou la nature des relations avec les parties prenantes.
\end{abstract}

Mots-clés : Entreprise sociale, Entrepreneuriat social, RSE

\section{Introduction}

La notion d'entreprise sociale creuse son sillon au sein de l'Union européenne. En témoigne la Communication de la Commission du 25 octobre 2011 concernant l'initiative pour l'entrepreneuriat social (Social Business ou Social Enterprise dans les versions anglaises). Des recherches internationales ont tenté de définir le concept d'entreprise sociale. Deux recherches font référence en la matière par l'ampleur des travaux. Celle de l'OCDE tout d'abord, puis celle du réseau européen EMES. L'entreprise sociale est ainsi vue comme une notion polysémique, véhiculée par différentes écoles (DEFOURNY, 2010).

L'entreprise sociale fait référence à toute activité privée d'intérêt général, organisée à partir d'une démarche entrepreneuriale, et n'ayant pas comme raison principale la maximisation des 
profits, mais la satisfaction de certains objectifs économiques et sociaux, ainsi que la capacité de mettre en place, par la production de biens ou de services, des solutions innovantes aux problèmes sociaux.

L'entreprise sociale a une finalité sociale ce qui met la finalité économique au second plan, celui d'un moyen mis à son service. Combinant des enjeux à la fois économiques et sociaux, ce type de structure mêle de multiples logiques d'action. Le caractère paradoxal des entreprises sociales contribue à mettre en relation deux mondes difficiles à réunir : le monde marchand, le monde du « business », et celui des services désintéressés rendus à une collectivité. D’où le nom parfois utilisé de « social business ».

L'entreprise sociale défendue par l'ESSEC et Ashoka, considère que les associations et les fondations reposant sur un modèle économique plutôt non marchand (répondant aux besoins de populations non solvables, menant des actions de plaidoyers ou de sauvegarde de l'environnement...), mais adoptant une démarche entrepreneuriale et veillant à leur pérennité économique, notamment en imaginant des modèles de financement innovants et en diversifiant leurs sources de financement, comptent parmi les « entreprises sociales », et sont bien dirigées par des « entrepreneurs sociaux».

Le concept de la RSE a connu une évolution extensive depuis sa diffusion aux différents volets. Née des préoccupations éthiques et morales autour des relations entrepreneuriales, la notion s'est étendue au gré des générations pour développer les finalités stratégiques de la firme et son rôle dans l'organisation. Ensuite sous la pression des PP, la RSE a adopté un plan pragmatique en développant des démarches de sensibilité sociale, ces derniers doivent permettre à l'entreprise de donner suite aux exigences de la société et des PP. Aujourd'hui, l'amélioration des outils de mesure de performance a intégré la RSE dans une nouvelle étape de mesure et de contrôle, la finalité étant de mesurer la performance sociale de l'entreprise et de vérifier sa corrélation avec sa performance globale.

Notre recherche consiste à présenter un état des lieux des entreprises sociales, entrepreneuriat social et RSE, mais également identifier les actions potentielles des entreprises classiques socialement responsables en matière de la RSE qui peuvent constituer un langage commun entre ces deux. D'où la formulation de notre problématique en la question principale suivante :

\section{«Dans quelles mesures la RSE permet-elle un langage commun entre les entreprises sociales et les entreprises classiques? »}


À partir de cette problématique centrale, nous déclinons un certain nombre de questionnements auxquels nous tenterons de répondre tout au long de cette étude :

- En quoi consiste la Responsabilité sociale des entreprises ?

- En quoi consistent les entreprises sociales ?

- Quels sont les principes fondamentaux des entreprises sociales?

- Quel lien existe-t-il entre les entreprises sociales et la RSE ?

- Quel lien existe-t-il entre entreprise sociale et entreprise classique socialement responsable?

L'objectif principal de cette recherche est de mettre en lumière les pratiques RSE adoptées par les entreprises classiques et leurs liens avec les principes fondamentaux des entreprises sociales. Dans le but d'atteindre cet objectif principal, notre analyse s'effectuera en deux temps :

Dans un premier temps, notre travail cherchera à présenter un état des lieux de l'ampleur de l'entrepreneuriat sociale, puis l'entreprise sociale et enfin la responsabilité sociale et la manière par laquelle elle doit être adoptée et déployer au sein de la structure organisationnelle des entreprises classiques.

Puis dans un second temps, on va s'arrêter sur un regard croisé sur les principes d'entreprise sociale et celles de la RSE.

Finalement, on procédera à une projection des pratiques RSE face au fondement des entreprises sociales, ce qui constituera le fruit de notre article.

\section{Cadre conceptuel}

La Responsabilité sociale des Entreprises (RSE) est une notion complexe qui s'est largement développée dans le monde entier. Connu depuis son origine éthique en Amérique, le concept a évolué pour inclure d'autres aspects économiques, managériaux, environnementaux, sociaux et politiques. Le fruit de cette évolution est une notion complexe qui ne se pratique pas de la même manière dans les pays du monde, compte tenu des différents facteurs environnementaux tels que la culture, les valeurs éthiques, la vision, la finalité de la nation ou la nature des relations avec les parties prenantes.

\subsection{Vers une définition d'entreprise sociale}

L'expression d'économie sociale apparaît pour la première fois au début du XXe siècle et précisément en 1912, dans un ouvrage intitulé "les institutions du progrès social" dont l'auteur 
est Charle GIDE. Dès le milieu des années 70, et surtout à partir de 1980 (début d'application des programmes d'ajustement structurel dans plusieurs pays), on assiste à l'émergence d'un ensemble de pratiques de l'économie solidaire.

\subsection{1. Émergence d'entrepreneuriat sociale}

Officialisé à travers le monde durant les 20 dernières années, le concept d'entrepreneuriat social se retrouve actuellement au cœur des débats portés sur le social. L'Europe et les ÉtatsUnis constituent notamment les principaux pionniers de cette conceptualisation.

\section{Approche anglo-saxonne}

C'est au début des années 1990 que la notion d'entrepreneuriat social signe son intégration officielle aux États-Unis. Différentes organisations et grandes universités, dont celles de New York, Columbia, et de Yale, sont les principaux initiateurs de l'émergence du concept. Dans le cadre de cette diversité conceptuelle, deux écoles ont été identifiées :

L'école de l'innovation sociale: L'accent est principalement mis sur les qualités de l'entrepreneur social, leadership, dynamisme, capacité d'apprentissage, d'adaptation, ou encore créativité, tous les critères entrent en jeu dans la concrétisation des projets sociaux.

L'école des ressources marchandes : Cette école développe des conceptions diverses, selon la génération de travaux concernée. Ainsi, une première division définit la notion d'entreprise sociale par l'ensemble des opérations économiques marchandes réalisées par les institutions privées à but non lucratif, dans l'objectif est de satisfaire les besoins sociaux.

\section{$>$ Approche européenne}

La naissance du concept d'entrepreneuriat social se trouve en Italie. En 1991, l'Italie a créé un statut spécifique de coopératives de solidarités sociales qui est la première référence européenne explicite à l'entrepreneuriat social. Quelques années après l'apparition du statut de « coopérative sociale » en Italie, de nombreux pays européens se retrouvent déjà en pleine concrétisation du concept. En 2004, le Royaume-Uni décide notamment de se pencher un peu plus sur le côté social, et crée une «cellule entreprise sociale» dans le but d'apporter plus de lumières aux entreprises sociales. La Belgique contribue également à l'expansion du concept d'entrepreneuriat social en permettant, à partir de l'année 1995, la qualité de « société à finalité sociale » aux entreprises pourtant commerciales.

\subsubsection{Conceptualisation d'entreprise sociale}


Une entreprise sociale est définie comme étant une entreprise dont le but est de générer un bénéfice social ou environnemental et non pas des plus-values pécuniaires, ses actionnaires savent bien qu'ils ne recevront pas de dividendes de leur placement, les employées du social business sont salariées et s'ils touchent des primes ou bonus, ceux-ci sont en fonction du bénéfice sociale qu'ils ont généré. Il existe plusieurs formes d'entreprises sociales à savoir : la coopérative, la fondation, la mutuelle, l'association.

\section{Les associations :}

Les associations sont régies par le dahir $\mathrm{n}^{\circ}$ 1-58-376 des 3 joumada I 1378 (15 novembre 1958) qui règlemente le droit d'association. Ce texte a subi de nombreuses modifications à travers des Dahirs ou des Décrets. Il définit l'association comme étant « un regroupement de personnes physiques et/ou de personnes morales sur une base contractuelle à durée déterminée ou à durée indéterminée ». Ces personnes mettent en commun, bénévolement et dans un but non lucratif, leurs connaissances et leurs moyens pour promouvoir et encourager les activités dans les domaines, notamment, professionnel, social, scientifique, religieux, éducatif, culturel, sportif, environnemental, caritatif et humanitaire.

L'association n'a pas automatiquement la personnalité juridique, pour cela elle doit faire l'objet d'une publicité officielle (annonce au Journal officiel). Il existe un débat sur l'appartenance de toutes les associations au secteur de l'économie sociale. En France un texte de $1981^{1}$, ne considère comme faisant partie de ce secteur, que les seules associations dites « gestionnaires », c'est-à-dire celles qui ont des activités de production ou de distribution de biens et de services marchands ${ }^{2}$. Or cette restriction, qui vise à exclure les activités associatives non marchandes ne paraît pas pertinente. En effet les associations non marchandes produisent de fait exclusivement de l'utilité sociale de façon directe, mais contribuent indirectement à la production d'utilité économique.

\section{Les coopératives :}

Le tissu coopératif constitue la principale composante du secteur de l'ESS au Maroc, tant par le nombre d'emplois créés que par sa participation à l'inclusion sociale et au développement

\footnotetext{
${ }^{1}$ Décret du 15 décembre 1981 instituant la délégation à l'économie sociale depuis supprimée

${ }^{2}$ Ce qui signifie que l'on instaure une distinction entre des activités qui produisent respectivement de l'utilité économique et de l'utilité sociale, et celles qui ne produisent « apparemment » que de l'utilité sociale
} 
économique. À cet effet, un cadre juridique lui est dédié : la loi $\mathrm{n}^{\circ} 112.12$ définit les coopératives, fixe leur statut juridique et établit les missions de l'ODCO. Ainsi ce sont des organisations qui correspondent aux caractéristiques suivantes : «Une coopérative est une association autonome de personnes volontairement réunies pour satisfaire leurs aspirations et besoins économiques, sociaux et culturels communs au moyen d'une entreprise dont la propriété est collective et où le pouvoir est exercé démocratiquement $»^{3}$.

«La coopérative est un groupement de personnes physiques et /ou morales, qui conviennent de se réunir pour créer une entreprise, leur permettant la satisfaction de leurs besoins économiques et sociaux, et qui est gérée conformément aux valeurs et principes fondamentaux mondialement reconnus en matière de coopération $»^{4}$.

\section{Les mutuelles :}

C'est le Dahir n¹-57-187 du 24 Joumada II 1383 (12 novembre 1969) qui définit les mutuelles et précise leurs champs d'activité et leurs objectifs. Ce même Dahir explique le rôle de la société mutuelle, de ses organes et leurs modes de fonctionnement. Au Maroc, le tissu mutualiste est constitué d'une cinquantaine d'institutions qui se répartissent principalement entre les mutuelles de santé et les mutuelles d'assurance. Alors, une mutuelle est un groupement de personnes morales de droit privé à but non lucratif, dont l'adhésion est volontaire. L'objectif est de proposer à ses membres, les sociétaires, un système de prévoyance, d'entraide et de solidarité, moyennant une cotisation. Selon le code de la mutualité, une mutuelle a pour objet "le développement culturel, moral, intellectuel et physique de ses membres et l'amélioration de leurs conditions de vie". Ce statut juridique est particulièrement fréquent dans le secteur de la santé.

\section{Les fondations :}

Une fondation est une personne morale de droit privé à but non lucratif créé par un ou plusieurs donateurs, eux-mêmes pouvant être des personnes physiques ou morales, pour accomplir une œuvre d'intérêt général. Les fondations sont rattachées à la famille des structures composant l'économie sociale. Le terme de fondation est défini par l'article 18 de la loi du 23 juillet 1987 : «Une fondation désigne l'acte par lequel une ou plusieurs personnes physiques

\footnotetext{
${ }^{3}$ Alliance coopérative internationale

${ }^{4}$ Selon la loi $\mathrm{N}^{\circ}$ 112-12 relative aux coopératives, publier au bulletin officiel en 2016.
} 
ou morales décident l'affectation irrévocable de biens, droits ou ressources à la réalisation d'une œuvre d'intérêt général et à but non lucratif ». Cette loi ajoute qu'une fondation «ne jouit de la capacité juridique qu'à compter de la date d'entrée en vigueur du décret en Conseil d'État accordant la reconnaissance d'utilité publique. Elle acquiert alors le statut de fondation reconnue d'utilité publique ».

\subsubsection{Principes fondamentaux d'entreprise sociale}

Le principal effort de conceptualisation de l'entreprise sociale provient d'un réseau de centres de recherche et de chercheurs indépendants baptisé « EMES $^{5}$ » (dont le Centre d'économie sociale de l'Université de Liège et le Centre Interdisciplinaire de Recherche Travail, État et Société (CIRTES) de l'Université Catholique de Louvain). En observant diverses initiatives d'entrepreneuriat social au sein de l'Union européenne et du reste du monde, EMES est parvenu à dégager neuf caractéristiques principales que 1'on retrouve en tout ou partie dans chaque entreprise sociale. Elles se répartissent en trois sous-ensembles :

Les indicateurs de nature économique : pour appréhender le caractère économique et entrepreneurial des initiatives envisagées, trois éléments ont été retenus :

Une activité continue de production de biens et/ou de services : l'activité productive représente la raison d'être - ou l'une des principales raisons d'être - des entreprises sociales. À l'inverse de certaines organisations non-profit traditionnel, les entreprises sociales n'ont pas comme activité principale la défense d'intérêts, ni la redistribution d'argent.

Un niveau significatif de prise de risque économique : Les créateurs d'une entreprise sociale assument totalement ou partiellement le risque qui y est inhérent.

Un niveau minimum d'emploi rémunéré : Tout comme les organisations non-profit traditionnel, les entreprises sociales peuvent faire appel à des ressources tant monétaires que non monétaires, et à des travailleurs rémunérés comme à des volontaires.

Les indicateurs de nature sociale : pour caractériser la dimension sociale des initiatives, trois indicateurs ont été privilégiés :

\footnotetext{
${ }^{5}$ EMES existe depuis 1996, est un réseau réunissant des centres de recherche universitaires et des chercheurs individuels reconnus, dont l'objectif est de construire progressivement un corpus européen de connaissances théoriques et empiriques sur l'économie sociale et l'entrepreneuriat social
} 
Un objectif explicite de service à la communauté : L'un des principaux objectifs des entreprises sociales est le service à la communauté ou à un groupe spécifique de personnes.

Une initiative émanant d'un groupe de citoyens : Les entreprises sociales résultent d'une dynamique collective impliquant des personnes qui appartiennent à une communauté ou à un groupe qui partage un besoin ou un objectif bien défini.

Un pouvoir de décision non basé sur la détention de capital : Ce critère renvoie généralement au principe "un membre, une voix", ou tout au moins à un processus de décision dans lequel les droits de vote au sein de l'assemblée détenant le pouvoir de décision ultime ne sont pas répartis en fonction d'éventuelles participations au capital.

Les indicateurs de la structure de gouvernance : pour caractériser la dimension de la structure de gouvernance, trois indicateurs ont été privilégiés :

Un degré élevé d'autonomie : Les entreprises sociales sont créées par un groupe de personnes sur base d'un projet propre et elles sont contrôlées par ces personnes. Elles peuvent dépendre de subsides publics, mais ne sont pas dirigées, que ce soit directement ou indirectement, par des autorités publiques ou d'autres organisations (fédérations, entreprises privées...).

Une dynamique participative, impliquant différentes parties concernées par l'activité : La représentation et la participation des usagers ou des clients, l'exercice d'un pouvoir de décision par diverses parties prenantes au projet et une gestion participative constituent souvent des caractéristiques importantes des entreprises sociales (promouvoir la démocratie).

Une limitation de la distribution des bénéfices : Si les entreprises sociales peuvent être des organisations caractérisées par une obligation absolue de non-distribution des bénéfices, elles peuvent aussi être des organisations qui, comme les coopératives dans beaucoup de pays, ont le droit de distribuer des bénéfices, mais de manière limitée.

\section{2. État des lieux de la RSE au sein des entreprises}

La RSE est considérée comme un ensemble des politiques, des pratiques et de leurs résultats et conséquence sur la protection de l'environnement et sur l'amélioration de la vie de la communauté (Tahri, 2014).

\subsubsection{Structuration de la démarche RSE}

La Responsabilité sociétale ou sociale d'une Entreprise ou Organisation (RSE/RSO) est la contribution des entreprises aux enjeux du développement durable, ainsi formaliser une 
démarche RSE permet d'évaluer, de structurer et de valoriser les engagements. Plusieurs études démontrent les intérêts d'une démarche RSE. La démarche RSE s'articule autour des 7 questions centrales de la norme ISO 26000, à savoir : la contribution au développement local, la protection des clients, les droits de l'Homme, les pratiques commerciales, l'environnement, les relations et conditions de travail, la gouvernance de l'Organisation.

Bien que les entreprises soient de plus en plus nombreuses à s'intéresser à la démarche RSE, sa mise en place peut paraître compliquée. Voici les étapes à respecter pour formaliser celle de son entreprise de manière efficace.

$>$ Étape 1 : Identifier les parties prenantes : Il s'agit de savoir qui est concerné par les actions de l'entreprise, d'identifier ceux dont les intérêts sont affectés par ses décisions.

> Étape 2 : Définir les attentes de la démarche : une fois qu'on a identifié les parties prenantes, il est nécessaire de comprendre leurs attentes et leurs besoins afin de pouvoir composer avec.

> Étape 3 : Déceler les enjeux RSE de l'entreprise : afin de bien cerner les enjeux, il faut s'intéresser aux opportunités et aux menaces que créeraient des mutations de marché et aux forces et faiblesses de l'entreprise.

> Étape 4 : Définir des objectifs qualitatifs et quantitatifs d'amélioration : à partir de ces enjeux, il va falloir définir de manière très précise les objectifs sociaux et environnementaux que l'on va se fixer.

> Étape 5 : Faire l'état des lieux actuel des performances durables de l'entreprise : pour avoir une idée nette du travail à fournir lors de la démarche, il faut chercher à observer et analyser la situation actuelle de la société, ses répercussions sociales et environnementales.

$>$ Étape 6 : Sensibiliser les parties prenantes à la démarche $R S E$ : les avantages concrets pour les parties prenantes doivent ensuite leur être exposés afin de les sensibiliser.

> Étape 7 : Former les collaborateurs à la démarche $\mathrm{RSE}$ : après l'observation et l'analyse des premières étapes, il va falloir mettre en place un plan d'action. Pour cela, les collaborateurs doivent être formés à la démarche qui va être entreprise.

$>$ Étape 8 : Communication interne et externe sur les engagements et la performance $\boldsymbol{R S E}$ : il faut ensuite faire connaître les valeurs que va afficher votre entreprise, les pratiques responsables qu'elle vise à encourager. 
> Étape 9 : Implication des salariés dans la démarche : les salariés doivent être particulièrement sensibilisés à la démarche.

> Étape 10 : Mise en place des recommandations par des actions : la dernière étape consiste à mettre en application les actions concrètes décidées lors de l'élaboration du plan d'action.

\subsubsection{Composition des pratiques RSE}

Les pratiques de la RSE sont distinguées par Vives (2006). Il s'agit des pratiques sociales internes (le personnel et l'environnement du travail) et/ou externes en visant la communauté et les pratiques environnementales relatives à la réduction de l'impact environnemental (veiller à réduire la consommation en énergie, recycler les déchets et utiliser les fournitures recyclées) ou encore les processus d'écoconception ou il s'agit de mettre en place le système de management environnemental, particulièrement dans les secteurs industriels qui ont un impact sur l'environnement (Paradas, 2006).

L'engagement dans une démarche socialement responsable se décline dans des pratiques que l'on peut qualifier de bonnes pratiques qui touchent les différents domaines de celle-ci, à savoir, le domaine économique, le domaine environnemental et le domaine social.

Les bonnes pratiques économiques : elles ne concernent pas uniquement la performance financière, mais aussi extra financière. Certaines pratiques socialement responsables semblent caractériser l'atteinte de cet objectif, il s'agit du respect des principes de saine concurrence, la lutte contre la corruption, l'intégration de l'éthique dans les actions commerciales, ainsi que la capacité de l'entreprise à contribuer au développement économique de sa zone d'implantation. Les bonnes pratiques environnementales : elles correspondent aux pratiques qui représentent toutes les actions entreprises afin de préserver l'environnement naturel.

Les bonnes pratiques sociales : elles englobent toutes les répercussions de l'activité de l'entreprise sur l'ensemble de ses parties prenantes ; fournisseurs, clients (sécurité et impacts psychologiques de produits), communautés locales (nuisances, respect des cultures) ; employés (conditions de travail, niveau de rémunération, non-discrimination, etc.), et la société en général.

\section{Regards croisés sur les entreprises sociales et la RSE}


Selon certains, les entreprises sociales entretiennent d'emblée, par leurs structures, leurs principes et les valeurs qui les sous-tendent, des affinités naturelles avec les objectifs du développement durable et partant, de la RSE, qui en est le pendant managérial.

\subsection{La conciliation entre principes des entreprises sociales et pratiques de la RSE}

Les entreprises sociales sont naturellement socialement responsables. En effet, les valeurs et principes sur lesquels elles sont fondées induisent par nature une attitude socialement responsable, soucieuse des répercussions sociales de leurs activités.

Ainsi l'intérêt général, le développement local et la cohésion sociale sont des valeurs défendues par les entreprises de l'ESS qui illustrent bien leurs engagements. Si les structures de l'économie sociale et solidaire sont par nature socialement responsables, il existe donc certains « éléments liants » avec la RSE. En regroupant plus en détail les spécificités des entreprises sociales et de la RSE, on obtient le tableau suivant :

Tableau : Caractéristiques comparées entre entreprises sociales et la RSE

\begin{tabular}{|c|c|}
\hline Entreprises sociales & Responsabilité sociale des entreprises \\
\hline Réponse de l'entreprise a un problème social & $\begin{array}{c}\text { Prise en compte des impacts des activités de } \\
\text { l'entreprise sur la société }\end{array}$ \\
\hline $\begin{array}{l}\text { Civique centrée sur le bien collectif, la } \\
\text { conscience sociale, la démocratie et le } \\
\text { renoncement à l'individualisme }\end{array}$ & $\begin{array}{l}\text { Commercial centrée sur la concurrence, l'intérêt } \\
\text { personnel et l'opportuniste }\end{array}$ \\
\hline Groupement volontaire de personnes & $\begin{array}{c}\text { La RSE doit être adoptée volontairement par } \\
\text { l'entreprise }\end{array}$ \\
\hline Actions volontaires & $\begin{array}{c}\text { Les mesures prises doivent dépasser les } \\
\text { exigences légales }\end{array}$ \\
\hline $\begin{array}{c}\text { Actions fondées sur des valeurs et principes } \\
\text { humanistes }\end{array}$ & $\begin{array}{l}\text { L'entreprise doit intégrer la RSE a son mode de } \\
\text { gestion de management }\end{array}$ \\
\hline $\begin{array}{l}\text { Participation des membres à la gouvernance } \\
\text { (relation avec les autres parties prenantes) }\end{array}$ & $\begin{array}{c}\text { La mise en place de la RSE ne peut se faire sans } \\
\text { un dialogue avec tous les parties prenantes }\end{array}$ \\
\hline $\begin{array}{c}\text { Non lucrativité } \\
\text { Absence de part sociale ou d'actions } \\
\text { Autonomie de gestion }\end{array}$ & $\begin{array}{l}\text { La RSE ne peut pas être motivée exclusivement } \\
\text { par la recherche de profits économiques }\end{array}$ \\
\hline $\begin{array}{c}\text { Recherche de la satisfaction de ses membres } \\
\text { Adaptation au changement }\end{array}$ & Adaptation au changement \\
\hline Solidarité Liberté d'adhésion & $\begin{array}{c}\text { L'entreprise doit intégrer des préoccupations } \\
\text { sociales }\end{array}$ \\
\hline $\begin{array}{l}\text { Actions concrètes, bilan sociétal, rapports } \\
\text { sociaux }\end{array}$ & $\begin{array}{l}\text { Une pratique de RSE doit pouvoir être évaluée } \\
\text { et vérifiée }\end{array}$ \\
\hline
\end{tabular}

${ }^{6}$ https://www.pourlasolidarite.eu 
On s'aperçoit ainsi qu'il existe de vrais liens sous-jacents entre RSE et entreprise sociale. Ces liens se recoupent dans les principes du développement durable. Les structures des entreprises sociales auraient dès lors tout intérêt à utiliser la RSE comme langage commun afin de dialoguer avec les entreprises classiques.

\subsection{La projection des pratiques RSE face au fondement des entreprises sociales}

Voyons maintenant comment la RSE se mesure au regard des principes des entreprises sociales, qui deviendront des critères d'évaluation de ces pratiques. Si les frontières entre les entreprises classiques et les entreprises sociales deviennent de plus en plus poreuses à mesure que celles-ci s'engagent dans des processus éthiques. Afin de structurer cette comparaison, nous prenons appui sur la typologie élaborée par Martinet et Payaud (2008), qui offre l'avantage de regrouper la diversité des pratiques de RSE en quatre niveaux d'engagement émanant des initiatives de RSE : la RSE cosmétique, périphérique, intégrée et Bottom of the Pyramid (BoP).

Les caractéristiques de ces quatre types d'initiatives permettent une comparaison entre certaines logiques d'inclusion de la RSE dans l'entreprise privée, d'une part, et les principes des associations, d'autre part.

\subsubsection{RSE cosmétique et périphérique}

Les deux premiers niveaux, la RSE cosmétique et périphérique, permettent peu de points de contact entre l'entreprise privée capitaliste et l'entreprise sociale. Le premier désigne la pratique la plus légère de la RSE. Ces initiatives se contentent de respecter des conditions légales et mettent en œuvre des projets minimaux et ponctuels et de degrés variables d'engagement.

Le second niveau, la RSE périphérique, caractérise les actions ayant un plus grand degré d'engagement, sans toutefois être incorporées à l'ensemble des pratiques de gestion. Il s'agit d'activités qui auraient pu être menées par n'importe quelle autre entreprise, puisque « la nature de l'action ne dépend pas des compétences de métier ou des activités de l'entreprise » (Martinet et Payaud, 2008 : 202), et qui sont, dans les faits, souvent laissés entre les mains d'un tiers ou d'une filiale (par exemple, une fondation) par l'intermédiaire de partenariats ou de subventions.

\subsubsection{RSE intégré}

Certaines entreprises vont toutefois plus loin et intègrent la RSE à leurs pratiques de gestion et à leurs activités courantes. Les initiatives relevant de la RSE intégrée sont en relation 
directe avec les activités de l'entreprise, que ce soit dans la gouvernance (par exemple, comité RSE au sein du conseil d'administration ayant un statut qui équivaut à celui des comités financiers) ou les activités mises en œuvre (par exemple, équipe d'intervention humanitaire liée au secteur d'activité de l'entreprise).

Une autre manière d'intégrer la RSE au cœur de l'entreprise est d'inclure des parties prenantes externes dans les décisions stratégiques. Éric Persais (2012) va jusqu'à proposer qu'une telle pratique atténue l'exclusivité du droit de propriété sur le capital, tout en apportant une validation sociale à cette propriété. De ce point de vue, certaines « entreprises capitalistes socialement responsables » (ECSR) intégreraient des pratiques de participation aux processus décisionnels au point de rejoindre les entreprises sociales sur ce plan.

Enfin, la rentabilité sociale visée par les entreprises sociales, qui se veut porteuse d'un projet politique d'économie alternative, est niée par l'entreprise capitaliste socialement responsable, qui, nous semble-t-il, cherche au contraire à permettre à la logique de l'entreprise privée de se maintenir dans un contexte où les parties prenantes externes sont de plus en plus informées, mobilisées et actives.

\subsubsection{RSE Botton of Pyramid}

Penchons-nous maintenant sur un quatrième type d'initiative de RSE : l'engagement social de certaines entreprises, qui se tournent vers les quatre milliards d'individus dans le monde qui vivent avec moins de deux dollars par jour, constituant le Bottom of the Pyramid (BoP). Ce « mouvement » entrepreneurial est considéré par Martinet et Payaud (2008) comme une forme particulièrement engagée de RSE. Son pari est de repenser l'opposition entre intérêt individuel et intérêt collectif, entre capitalisme et philanthropie. Ce type d'initiative est à l'œuvre lorsque des entreprises privées capitalistes mettent en avant des innovations radicales, du point de vue de l'offre, des moyens de production ou des méthodes de distribution, pour offrir aux plus pauvres des produits et des services, souvent de première nécessité, qui leur étaient jusque-là inaccessibles. Il s'agit ainsi de voir les plus pauvres comme une « occasion d'affaires », tout en s'appuyant sur des partenariats avec les populations locales, ce qui permet de « redessiner totalement le système d'activités et de le rendre économiquement viable » (Payaud, $2012: 3$ ).

Sans nier les avantages de cet engagement sociétal des entreprises pour les populations les plus pauvres, nous devons souligner que le projet social, les principes et les critères 
fondamentaux des entreprises sociales reposent par nature sur l'aide sociale aux populations pauvres voire même les plus vulnérables.

\section{Conclusion}

Au terme de cette comparaison entre les pratiques RSE et les fondements des entreprises sociales, les frontières entre les entreprises sociales et les entreprises privées à fort engagement dans les pratiques de RSE semblent effectivement poreuses et floues. Si les principes et les structures caractérisant les entreprises sociales montrent des convergences certaines avec les principes de la RSE, ils ne constituent pas toujours des garanties de pratiques innovantes en matière de redistribution de la richesse, d'amélioration des conditions de travail des employés, ni de respect de l'environnement.

Par ailleurs, certains types d'engagement socialement responsable rapprochent l'entreprise privée capitaliste des entreprises sociales à certains égards, notamment lorsqu'ils permettent aux parties prenantes de prendre réellement part aux orientations de l'entreprise ou qu'ils proposent une redéfinition de l'aide internationale par une offre de produits et services adaptée aux besoins des individus les moins fortunés des pays en voie de développement.

Néanmoins, cette comparaison met également en évidence certaines caractéristiques des entreprises sociales, qui lui sont exclusives et la distinguent toujours de l'entreprise capitaliste, aussi socialement responsable et engagée soit-elle : la participation à un renouvellement de la vie démocratique, la propriété collective, l'inclusion des parties prenantes au sein même de l'effectif des membres, l'ancrage territorial et social d'un projet qui émane des besoins de la volonté d'un groupe d'individus, qui veulent se donner ensemble les moyens d'y répondre.

\section{BIBLIOGRAPHIE}

[1] «SD 21000 Développements durables responsabilité sociétale des entreprises », AFNOR, 2003 Saint Denis, $41 \mathrm{P}$

[2] «La diffusion de la RSE dans les entreprises au Maroc : Facteurs déterminants, rôles et interactions des acteurs. », Benaicha O., le 17 janvier 2017, thèse de doctorat, soutenue a Casablanca, $465 \mathrm{P}$

[3] « Perspective historique de la responsabilité sociale des entreprises (RSE) », Jbara N., vol 11, Meknès 2017, revue multidisciplinaire sur l'emploi le syndicalisme et le travail (REMEST), 17 P.

[4] « Audit social », Igalens J. et Peretti J. M., 2016, Paris, 2eme Édition Eyrolle, 192 P. 
[5] «Analyse des pratiques de responsabilité sociétale des entreprises », Mazari L., 2014, Montpelier, $94 \mathrm{P}$.

[6] « Audit social», Igalens J. et Peretti J. M., 2016, Paris, 2eme Édition Eyrolle, P 192.

[7] «Les associations au Maroc : cadre juridique », Brahimi M., 1998, publication du Centre de documentation des collectivités locales.

[8] «La diffusion de la RSE dans les entreprises au Maroc : Facteurs déterminants, rôles et interactions des acteurs. », Benaicha O., thèse de doctorat, soutenue à Casablanca le 17 janvier 2017, P 465.

[9] « Analyse des pratiques de responsabilité sociétale des entreprises », Mazari L., 2014, Montpelier, P 94.

[10] « Pratique RSE et performance globale : cas des associations de la région Fès-Meknès » Mustapha OUKASSI, Imad EL GHMARI, Omar EL GHMARI, p12

[11] « Réflexion sur l'état actuel de la responsabilité sociétale des entreprises au Maroc », El hila R. et Amaazoul H., 2011, Oujda, P 17.

[12] « l'importance de l'épistémologie pour la recherche en droit », Alban G., 2015, Presse de l'université, Toulouse 1 capitole, Lyon, P 40.

[13] «Épistémologie et méthodologie de la recherche en science de gestion. Note de synthèse », Velmuradova M., 2017, Toulon, Hal archives ouvertes, P105. 